【生物工程／Bioengineering】

\title{
新型近红外花菁类光声探针用于肼检测
}

\author{
蒋 超，林 静，黄 鹏 \\ 深圳大学医学部生物医学工程学院, 广东深圳 518060
}

摘 要: 肼含量的检测在化工、环境和生命健康等领域具有重要意义. 利用在近红外区域具有较强吸 收的花菁类分子为母体，发展新型光声探针用于肼检测. 该探针在波长 $808 \mathrm{~nm}$ 处具有很强的光声信号. 当 探针分子与肼作用后，随着溶液中肼浓度的增高，分子探针在波长 $808 \mathrm{~nm}$ 处的光密度 $D(808)$ 显著下降， 而 $550 \mathrm{~nm}$ 处的光密度 $D(550)$ 明显升高，且在㭌浓度为 $0 \sim 5 \mu \mathrm{mol} / \mathrm{L}$ 内, $D(808) / D(550)$ 比值随胖浓度变 化具有良好的线性关系。同时，新型光声探针在 $808 \mathrm{~nm}$ 处的光声信号强度 $P(808)$ 也随胖浓度增加而降 低，并且在肼浓度为 $0 \sim 10 \mu \mathrm{mol} / \mathrm{L}$ 内, $P(808)$ 与肼浓度呈良好的线性关系，肼检测限为 $0.50 \mu \mathrm{mol} / \mathrm{L}$. 该 探针合成方法简单，并能够对肼实现快速高效的光声检测，为今后在体胖的检测提供了一种新的方法.

关键词：生物医学工程；光声检测；光声成像；花菁类分子探针；肼检测；近红外光谱

中图分类号：Q819 文献标志码：A doi：10.3724/SP. J.1249.2020.01033

\section{Novel near-infrared cyanine-based photoacoustic probe for hydrazine detection}

\author{
JIANG Chao, LIN Jing, and HUANG Peng \\ School of Biomedical Engineering, Health Science Center, Shenzhen University, Shenzhen 518060, Guangdong Province, P. R. China
}

\begin{abstract}
Hydrazine detection is of great significance in the fields of chemical industry, environment, life, health, and so on. A novel photoacoustic probe is designed and synthesized for hydrazine detection, using cyanine with strong near-infrared absorbance as the core. The probe has strong photoacoustic signal at $808 \mathrm{~nm}$. When the probe molecules interact with the hydrazine, the absorbance intensities of probe at $808 \mathrm{~nm}$ and $550 \mathrm{~nm}$ decrease and increase with the increase of hydrazine concentration, respectively. The optical density ratio of $D(808) / D(550)$ in the range of $0-5 \mu \mathrm{mol} / \mathrm{L}$ hydrazine concentration has a good linear relationship with the change of concentration. Meanwhile, photoacoustic intensity of probe at $808 \mathrm{~nm} P(808)$ gradually decreases with the increase of hydrazine concentration, and shows a good linear relationship in the range of $0-10 \mu \mathrm{mol} / \mathrm{L}$, with a detection limit of 0.50 $\mu \mathrm{mol} / \mathrm{L}$. The probe is easy to synthesis and can achieve photoacoustic detection of hydrazine rapidly and efficiently, which provids a new solution for the development of in vivo detection of hydrazine.
\end{abstract}

Key words : biomedical engineering; photoacoustic detection; photoacoustic imaging; cyanine molecule probe; hydrazine detection; near infrared spectroscopy

肼 $\left(\mathrm{N}_{2} \mathrm{H}_{4}\right)$ 是一种常见的化工原料，是合成聚 合物、纺织染料和医药中间体等的重要前体 ${ }^{[1]}$. 肼
具有易燃易爆特点，被广泛应用于火箭燃料和重氮 燃料的生产中 ${ }^{[2]}$. 但是，肼对皮肤有很强的侵蚀作

Received: 2019-09-28; Accepted: 2019-10-29

Foundation: National Basic Research Program of China (2018YFA0704003)

Corresponding author: Professor HUANG Peng. E-mail: peng.huang@ szu.edu.cn

Citation: JIANG Chao, LIN Jing, HUANG Peng. Novel near-infrared cyanine-based photoacoustic probe for hydrazine detection $[\mathrm{J}]$. Journal of Shenzhen University Science and Engineering, 2020, 37(1):33-38. (in Chinese) 
用, 尤其对眼睛和肝脏等器官有严重的损伤作用, 被列为常见致癌物之一 ${ }^{[3-4]}$. 因此, 肼的检测对环 境保护和生命健康都具有重要意义.

目前, 关于肼的检测方法主要有库仑法 ${ }^{[5-6]}$ 、 电位滴定法 ${ }^{[7-8]}$ 和苂光检测法 ${ }^{[9-10]}$. 其中，库仑法 和电位滴定法操作复杂，检测时间长，且无法实现 活体生物组织检测 ${ }^{[11]}$; 苂光检测法因具有灵敏度 高、采集样品时间短和时间分辨率高的优点而被广 泛用于科学研究 ${ }^{[12]}$. 然而, 苂光成像的激发光波 长范围通常在 $400 \sim 700 \mathrm{~nm}$, 组织穿透深度有限, 对于深层组织无法检测, 并且生物组织自发苂光背 景较强, 严重干扰其在活体生物组织中的应 用 ${ }^{[13-14]}$. 因此, 找出一种实时、快速, 且可用于活 体深层组织肼检测的方法非常有意义.

光声检测 ( photoacoustic detection, PAD) 是一种 利用光声成像 (photoacoustic imaging, PAI) 对待测物 进行检测的新技术，主要基于光声（photoacoustic， PA）探针和检测物之间的相互作用，根据 PA 信号 的变化作为指标, 实现对待测物的检测 ${ }^{[15]}$. 与其 他成像方式相比, PAI 是一种新兴的无创、非电离 成像, 当脉冲激光射入生物组织时, 组织吸收脉冲 激光能量转化为热能, 引发热弹性膨胀, 从而产生 超声信号, 超声探头收集到超声信号并成像. PAI 结合了光学成像和超声成像两者优点, 具有对比度 丰富、分辨率高和深层组织穿透力强等优点 ${ }^{[16]}$. 因此, PAI 可提供深层组织一些特定目标的高分辨 率图像信息 ${ }^{[17]}$. 目前已开发出多种基于无机或有 机材料的 PAI 造影剂用于肿瘤和组织器官的成 像 ${ }^{[18-20]}$. 随着 PA 探针的发展，PAD 已被广泛应用 于肿瘤诊断 ${ }^{[21-22]}$ 、离子检测 ${ }^{[23-27]}$ 以及酶检测 ${ }^{[28-30]}$ 等 领域. 但是, 目前肼检测的光声探针还有待开发.

本研究提出了利用花菁类染料为母体，设计并 合成了一种新型 PA 分子探针 ( $\mathrm{Cy}-2)$, 成功实现了 对肼的有效检测.

\section{1 实 验}

\section{1 试 剂}

2-[2-[2-氯-3-[2-( 1, 3 二氢-1, 3, 3-三甲基 L2H-苯并吲哚-2-烯基) -乙烯基 ]-1-环己烷-1-基 ]-乙 烯基]-1,3,3-三甲基-1 $\mathrm{H}$-苯并吲哚鎓对甲苯磺酸盐 ( IR-813, $\mathrm{C}_{47} \mathrm{H}_{47} \mathrm{ClN}_{2} \mathrm{O}_{3} \mathrm{~S}$ )、 $\mathrm{N}, \mathrm{N}$-二甲基甲酰胺 (dimethyl formamide, DMF)、三乙胺 $\left(\mathrm{C}_{6} \mathrm{H}_{15} \mathrm{~N}\right.$ triethylamine, TEA )、丙烯酰氯 $\left(\mathrm{C}_{3} \mathrm{H}_{3} \mathrm{ClO}\right)$ 、硫酸钠
$\left(\mathrm{Na}_{2} \mathrm{SO}_{4}\right)$ 、硝酸钠 $\left(\mathrm{NaNO}_{3}\right)$ 、氯化铵 $\left(\mathrm{NH}_{4} \mathrm{Cl}\right)$ 、六水 合硝酸锌 $\left(\mathrm{Zn}\left(\mathrm{NO}_{3}\right)_{2} \cdot 6 \mathrm{H}_{2} \mathrm{O}\right)$ 、氯化永 $\left(\mathrm{HgCl}_{2}\right)$ 、氯 化镁 $\left(\mathrm{MgCl}_{2}\right)$ 、氯化铜 $\left(\mathrm{CuCl}_{2}\right)$ 、氯化锰 $\left(\mathrm{MnCl}_{2}\right)$ 、醋 酸铅 $\left(\left(\mathrm{CH}_{3} \mathrm{COO}\right)_{2} \mathrm{~Pb}\right)$ 和肼 $\left(\mathrm{N}_{2} \mathrm{H}_{4} \cdot \mathrm{H}_{2} \mathrm{O}\right)$ 均购自北 京百灵威科技有限公司; 乙酸钠 $\left(\mathrm{C}_{2} \mathrm{H}_{3} \mathrm{NaO}_{2}\right)$ 购自上 海萨恩化学科技有限公司; 二氯甲烷 $\left(\mathrm{CH}_{2} \mathrm{Cl}_{2}\right.$, dichloromethane, DCM) 和氯化钠 $(\mathrm{NaCl})$ 购自西格 玛-奥德里奇中国; 溴化钾 $(\mathrm{KBr})$ 、硝酸银 $\left(\mathrm{AgNO}_{3}\right)$ 和氯化钾 $(\mathrm{KCl})$ 购自上海麦克林生化科技有限公司. 以上所用试剂均为分析纯, 无特别说明时均未提纯 处理, 且所有溶液均由去离子水 (millipore, direct- $Q$ 3 超纯水系统) 配制.

\section{2 仪 器}

紫外-可见光分光光度计 (安捷伦科技 (中国) 有 限公司生产, 型号为 Cary 60 ) ; 小动物活体光声成 像系统 (型号为 Vevo LAZR2100, VisualSonics 富士 公司生产).

\section{3 合成步骤}

本研究合成的 Cy-2 探针由花菁染料 IR-813 衍 生物 $\mathrm{Cy}-1$ 与烯丙酰氯酯化反应得到, 合成步骤如 图 1.

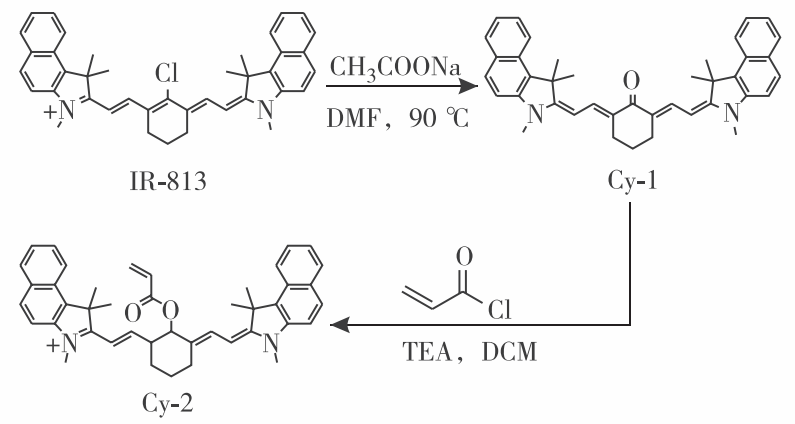

图 1 探针 Cy-2 的合成步骤

Fig. 1 The synthetic route of $\mathrm{Cy}-2$ probe

\subsection{Cy-1 的合成}

在氮气保护下，将化合物 IR-813 (305 mg, $0.50 \mathrm{mmol})$ 溶于无水 DMF $(10 \mathrm{~mL})$ 中, 然后加人 乙酸钠 $(123 \mathrm{mg}, 1.50 \mathrm{mmol})$. 反应混合液加热至 $90{ }^{\circ} \mathrm{C}$, 搅拌 $3 \mathrm{~h}$ 后, 冷却至室温. 除去溶剂, 粗产 物使用石油醚和乙酸乙酯 (体积比为 $3: 1$ ) 作为洗脱 剂洗脱，利用硅胶柱层析分离得到红色固体粉末 $\mathrm{Cy}-1,151 \mathrm{mg}$, 产率 $65 \%$.

\subsubsection{Cy-2 的合成}

在氮气保护下, 将化合物 Cy-1 (780 mg) 和 TEA $(170 \mathrm{mg})$ 溶于无水 DCM $(20 \mathrm{~mL})$ 中, 搅拌 15 $\min$, 然后在冰浴条件下逐滴加人丙烯酰氯 
$(456 \mathrm{mg})$ 的 DCM 溶液, 继续搅拌 $20 \mathrm{~min}$. 暖至室 温, 搅拌 $3 \mathrm{~h}$. 除去溶剂, 使用 DCM 和甲醇 (体积 比 30:1）作为洗脱剂洗脱, 利用硅胶柱层析分离得 到绿色固体粉末 $\mathrm{Cy}-2,392 \mathrm{mg}$, 产率 $42 \%$. 化合物 的氢 $\left({ }^{1} \mathrm{H}\right)$ 核磁共振谱 (nuclear magnetic resonance spectroscopy, NMR) (500 MHz, $\mathrm{CDCl}_{3}$ ) 化学位移 $\delta$ 分别为 $7.97 \sim 7.80(\mathrm{~m}, 41 \mathrm{H}), 7.79(\mathrm{~d}, J=2.4$ $\mathrm{Hz}, 15 \mathrm{H}), 7.39(\mathrm{~d}, J=30.9 \mathrm{~Hz}, 28 \mathrm{H}), 7.28(\mathrm{~s}$, $9 \mathrm{H}), 7.15(\mathrm{~s}, 9 \mathrm{H}), 6.92(\mathrm{~d}, J=7.5 \mathrm{~Hz}, 9 \mathrm{H})$, $6.53 \sim 6.33(\mathrm{~m}, 9 \mathrm{H}), 6.26(\mathrm{~s}, 9 \mathrm{H}), 6.06(\mathrm{dd}$, $J=14.7,8.1 \mathrm{~Hz}, 7 \mathrm{H}), 6.03 \sim 5.87(\mathrm{~m}, 12 \mathrm{H})$, $5.28(\mathrm{~s}, 19 \mathrm{H}), 5.05(\mathrm{~s}, 9 \mathrm{H}), 4.29(\mathrm{dd}$, $J=15.0,6.2 \mathrm{~Hz}, 9 \mathrm{H}), 3.30(\mathrm{~s}, 27 \mathrm{H}), 2.72 \sim$ $2.68(\mathrm{~m}, 9 \mathrm{H}), 2.59(\mathrm{~s}, 27 \mathrm{H}), 2.35 \sim 2.31(\mathrm{~m}$, $7 \mathrm{H}), 2.30 \sim 2.26(\mathrm{~m}, 7 \mathrm{H}), 1.83(\mathrm{~s}, 1 \mathrm{H}), 1.79$ $(\mathrm{d}, J=20.6 \mathrm{~Hz}, 64 \mathrm{H}), 1.70(\mathrm{~s}, 7 \mathrm{H}), 1.47(\mathrm{~d}$, $J=16.6 \mathrm{~Hz}, 63 \mathrm{H}), 1.39(\mathrm{~s}, 7 \mathrm{H})$. 电喷雾质谱 (electrospray ionization-mass spectrometry, ESI-MS ) : $[\mathrm{M}]^{+}=621.3423$ (计算得 $\mathrm{C}_{43} \mathrm{H}_{45} \mathrm{~N}_{2} \mathrm{O}_{2}{ }^{+}: 621.33$ ).

\section{4 溶液配制}

探针配制: 准确称取 $\mathrm{Cy}-2$ 探针 $24.84 \mathrm{mg}$ 溶解 于 $4 \mathrm{~mL}$ 的甲醇中, 终浓度为 $10 \mathrm{mmol} / \mathrm{L}$.

肼及各种离子溶液的配制: 通过将肼及各种离 子相应的盐分别为 $\mathrm{Na}_{2} \mathrm{SO}_{4} 、 \mathrm{NaNO}_{3} 、 \mathrm{NaCl} 、 \mathrm{KBr}$ 、 $\mathrm{CH}_{3} \mathrm{COONa} 、 \mathrm{NH}_{4} \mathrm{Cl} 、 \mathrm{NaCl} 、 \mathrm{KCl} 、 \mathrm{AgNO}_{3}$ 、 $\mathrm{Zn}\left(\mathrm{NO}_{3}\right)_{2} 、 \mathrm{HgCl}_{2} 、 \mathrm{MgCl}_{2} 、 \mathrm{CuCl}_{2} 、 \mathrm{MnCl}_{2}$ 或 $\left.\left(\mathrm{CH}_{3} \mathrm{COO}\right)_{2} \mathrm{~Pb}\right)$ 溶于去离子水中, 制备各种离子 溶液 $\mathrm{SO}_{4}{ }^{2-} 、 \mathrm{NO}_{3}^{-} 、 \mathrm{Cl}^{-} 、 \mathrm{Br}^{-} 、 \mathrm{CH}_{3} \mathrm{COO}^{-}$、 $\mathrm{NH}_{4}^{+} 、 \mathrm{Na}^{+} 、 \mathrm{~K}^{+} 、 \mathrm{Ag}^{+} 、 \mathrm{Zn}^{2+} 、 \mathrm{Hg}^{2+} 、 \mathrm{Mg}^{2+} 、$ $\mathrm{Cu}^{2+} 、 \mathrm{Mn}^{2+} 、 \mathrm{~Pb}^{2+}$ 和肼), 浓度均为 $3 \mathrm{~mol} / \mathrm{L}$, 备用.

\section{2 结果与分析}

\section{$2.1 \mathrm{Cy}-2$ 对肼的检测灵敏度研究}

为探究 Cy-2 对肼的光学响应, 采用紫外-可见 分光光度计测定不同浓度肼对 $\mathrm{Cy}-2$ 的吸收光谱影 响; 采用小动物光声成像系统测定加人不同浓度肼 后的 $\mathrm{Cy}-2$ 探针在 $808 \mathrm{~nm}$ 处光声强度 $P(808)$ 变化.

向 $\mathrm{Cy}-2(10 \mu \mathrm{mol} / \mathrm{L})$ 溶液中, 逐渐加入肼 $(0 \sim$ $10 \mu \mathrm{mol} / \mathrm{L})$, 测定不同浓度的肼对 $\mathrm{Cy}-2$ 溶液吸收 光谱的影响, 结果如图 2. 随着溶液中肼浓度的增 高, $\mathrm{Cy}-2$ 溶液体系在波长为 $808 \mathrm{~nm}$ 处的光密度 $D(808)$ 显著下降, 而 $550 \mathrm{~nm}$ 处的光密度 $D(550)$ 明显升高. 同时, 在肼浓度由 $0 \sim 10 \mu \mathrm{mol} / \mathrm{L}$ 变化
时, 伴有明显的颜色变化 (图 2 插图). 在肼浓度 为 $0 \sim 5 \mu \mathrm{mol} / \mathrm{L}$ 内, Cy-2 在 $808 \mathrm{~nm}$ 和 $550 \mathrm{~nm}$ 处的 光密度比值 $D(808) / D(550)$ 与肼浓度具有良好的 线性关系, 如图 3. 依据图 3 (b) 的线性关系, 可 以根据 $D(808) / D(550)$ 比值推算出待测物中肼的 浓度.

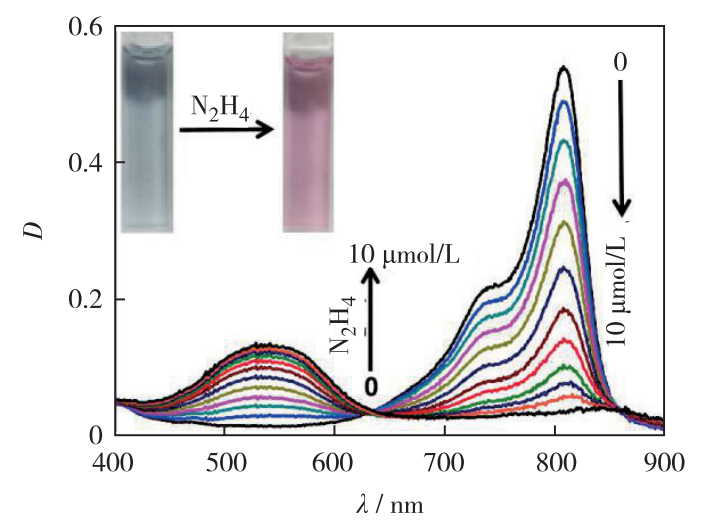

图 2 Cy-2 (10 $\mu \mathrm{mol} / \mathrm{L})$ 与不同浓度 $(0 \sim 10 \mu \mathrm{mol} / \mathrm{L})$ 肼反应后的吸收光谱图

Fig. 2 Absorption spectra of Cy-2 solutions ( $10 \mu \mathrm{mol} / \mathrm{L})$ after reacting with different concentrations of hydrazine $(0 \sim 10 \mu \mathrm{mol} / \mathrm{L})$

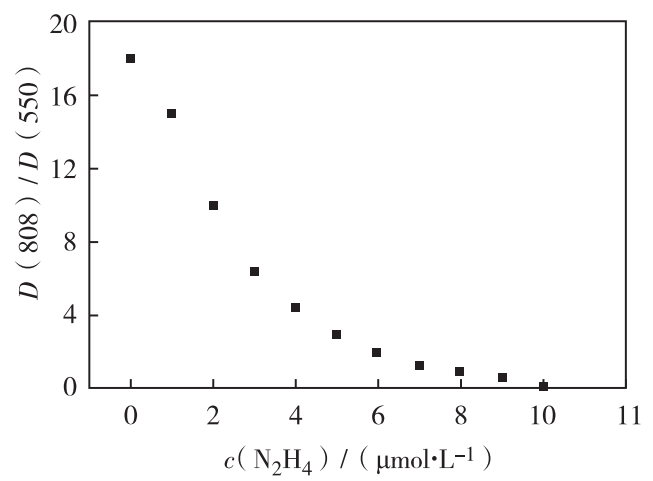

(a) $D(808) / D(550)$ 随肼浓度变化

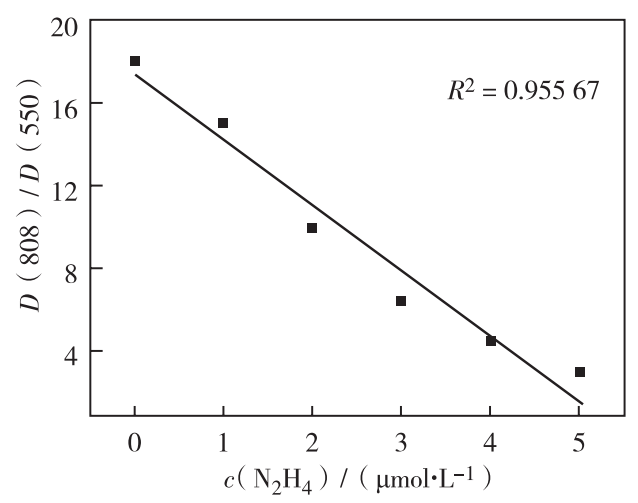

(b) $D(808) / D(550)$ 与肼浓度关系

图 3 Cy-2 与不同浓度肼反应后的 $D(808) / D(550)$

Fig. $3 D(808) / D(550)$ of Cy-2 against the concentration of hydrazine 
然后，测定不同浓度的肼对 $\mathrm{Cy}-2$ 溶液光声强 度的影响. 向 $\mathrm{Cy}-2(10 \mu \mathrm{mol} / \mathrm{L})$ 溶液中分别加人不 同浓度的肼 $(0 \sim 10 \mu \mathrm{mol} / \mathrm{L})$, 结果如图 4. 随着溶 液中肼浓度的增高, 探针的 $P(808)$ 明显下降, 且 在一定浓度内 $(0 \sim 10 \mu \mathrm{mol} / \mathrm{L}), P(808)$ 与肼浓度 具有良好的线性关系，如图 5 , 肼检测限为 0.50 $\mu \mathrm{mol} / \mathrm{L}$.

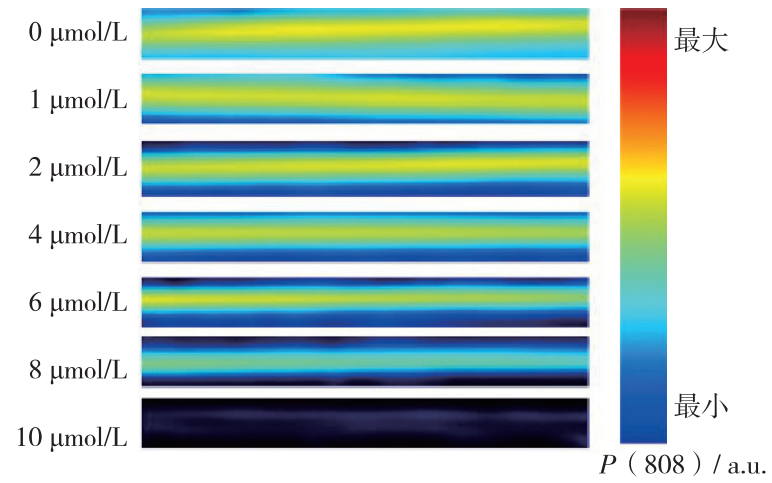

图 $4 \mathrm{Cy}-2$ 与不同浓度肼 $(0 \sim 10 \mu \mathrm{mol} / \mathrm{L})$ 反应后, 在 $808 \mathrm{~nm}$ 处光声图像

Fig. 4 PA images of Cy-2 in the presence of different concentrations of hydrazine at $808 \mathrm{~nm}$

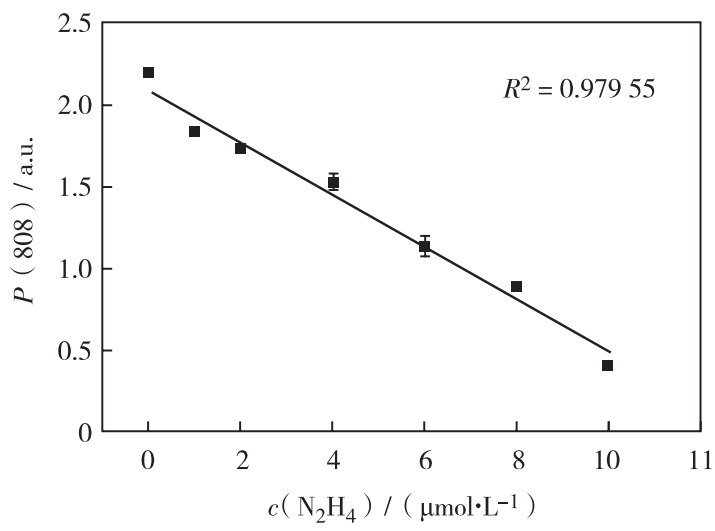

图 5 探针 $P(808)$ 与肼浓度呈线性关系

Fig. $5 P(808)$ of $\mathrm{Cy}-2$ against the concentration of hydrazine

\subsection{Cy-2 对肼的检测选择性研究}

为探究 Cy-2 对肼的选择性，利用紫外-可见光 谱和光声成像技术，共同验证探针 Cy-2 对肼的识 别特异性. 向 $\mathrm{Cy}-2(10 \mu \mathrm{mol} / \mathrm{L})$ 的甲醇溶液中, 分别加人 $100 \mu \mathrm{mol} / \mathrm{L}$ 的 $\mathrm{N}_{2} \mathrm{H}_{4} 、 \mathrm{NO}_{3}{ }^{-} 、 \mathrm{Cl}^{-} 、 \mathrm{Br}^{-}$、 $\mathrm{SO}_{4}{ }^{2-} 、 \mathrm{CH}_{3} \mathrm{COO}^{-} 、 \mathrm{NH}_{4}^{+} 、 \mathrm{Na}^{+} 、 \mathrm{~K}^{+} 、 \mathrm{Ag}^{+}$、 $\mathrm{Zn}^{2+} 、 \mathrm{Mg}^{2+} 、 \mathrm{Cu}^{2+} 、 \mathrm{Mn}^{2+} 、 \mathrm{Hg}^{2+}$ 和 $\mathrm{Pb}^{2+}$ ，待反应 $5 \mathrm{~min}$ 后，检测各反应溶液的吸收光谱，结果如图 6. 由图 6 可见，只有加人肼的溶液的吸收峰位置
发生显著的蓝移，在 $808 \mathrm{~nm}$ 处的吸收峰消失，在 $550 \mathrm{~nm}$ 处出现新的吸收峰, 而其他溶液的吸收光谱 几乎没有变化. 如图 7 所示，只有加人肼的 Cy-2 溶液颜色由原来的绿色变成了红色, $D(808) / D(550)$ 比值降低; 而加人其他离子的溶 液颜色及 $D(808) / D(550)$ 比值均无明显变化，表 明 Cy-2 对肼检测的特异性很好.

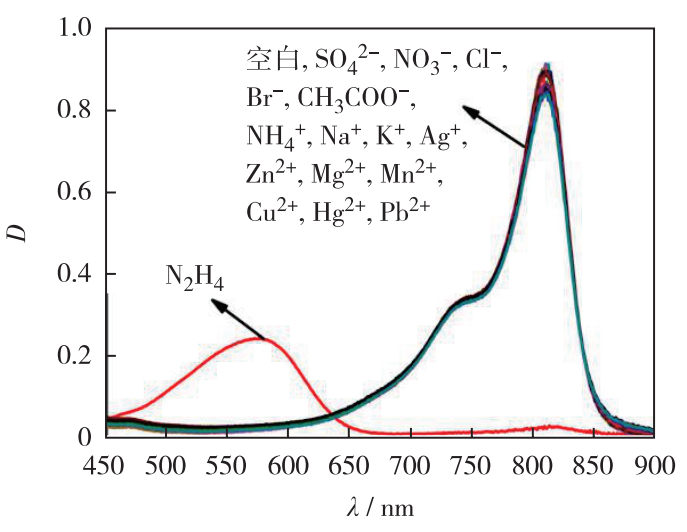

图 $6 \mathrm{Cy}-2$ 与 $100 \mu \mathrm{mol} / \mathrm{L}$ 的肼和 其他离子作用的吸收光图谱

Fig. 6 Absorption spectra of $\mathrm{Cy}-2$ with the addition of $100 \mu \mathrm{mol} / \mathrm{L}$ of hydrazine and other ions

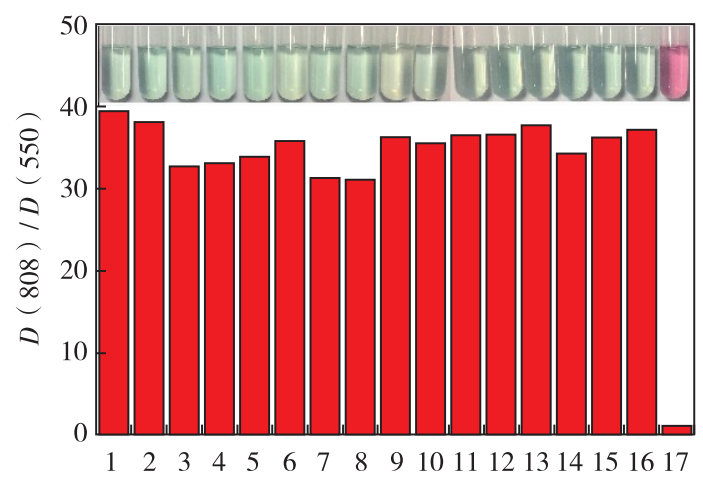

1 为 $\mathrm{Cy}-2 ; 2$ 为 $\mathrm{SO}_{4}{ }^{2-} ; 3$ 为 $\mathrm{NO}_{3}{ }^{-} ; 4$ 为 $\mathrm{Cl}^{-} ; 5$ 为 $\mathrm{Br}^{-}$; 6 为 $\mathrm{CH}_{3} \mathrm{COO}^{-} ; 7$ 为 $\mathrm{NH}_{4}{ }^{+} ; 8$ 为 $\mathrm{Na}^{+} ; 9$ 为 $\mathrm{K}^{+} ; 10$ 为 $\mathrm{Ag}^{+}$;

11 为 $\mathrm{Zn}^{2+} ; 12$ 为 $\mathrm{Hg}^{2+} ; 13$ 为 $\mathrm{Mg}^{2+} ; 14$ 为 $\mathrm{Cu}^{2+}$; 15 为 $\mathrm{Mn}^{2+} ; 16$ 为 $\mathrm{Pb}^{2+} ; 17$ 为 $\mathrm{N}_{2} \mathrm{H}_{4}$

图 7 Cy-2 分别与肼、其他离子 $(100 \mu \mathrm{mol} / \mathrm{L})$ 作用后 颜色及 $D(808) / D(550)$ 比值变化

Fig. 7 Color changes of Cy-2 towards $100 \mu \mathrm{mol} / \mathrm{L}$ of hydrazine and other ions and $D(808) / D(550)$ of Cy-2 in the presence of hydrazine and other ions

随后，利用光声成像来研究 $\mathrm{Cy}-2$ 对肼的识别 特异性. 向 $\mathrm{Cy}-2(10 \mu \mathrm{mol} / \mathrm{L})$ 的甲醇溶液中, 分 别加人 $100 \mu \mathrm{mol} / \mathrm{L}$ 的 $\mathrm{N}_{2} \mathrm{H}_{4} 、 \mathrm{NO}_{3}{ }^{-} 、 \mathrm{Cl}^{-} 、 \mathrm{Br}^{-}$、 
$\mathrm{SO}_{4}{ }^{2-} 、 \mathrm{CH}_{3} \mathrm{COO}^{-} 、 \mathrm{NH}_{4}^{+} 、 \mathrm{Na}^{+} 、 \mathrm{~K}^{+} 、 \mathrm{Ag}^{+} 、$ $\mathrm{Zn}^{2+} 、 \mathrm{Mg}^{2+} 、 \mathrm{Cu}^{2+} 、 \mathrm{Mn}^{2+} 、 \mathrm{Hg}^{2+}$ 和 $\mathrm{Pb}^{2+}$ ，待反应 $5 \mathrm{~min}$ 后, 测得各反应液的光声信号强度. 如图 8 和图 9, 只有加人肼溶液的探针在 $808 \mathrm{~nm}$ 处的光声 信号强度下降，而其他离子的加人都不会引起明显 变化, 实验结果再次表明 $\mathrm{Cy}-2$ 对肼具有很好的选 择性.

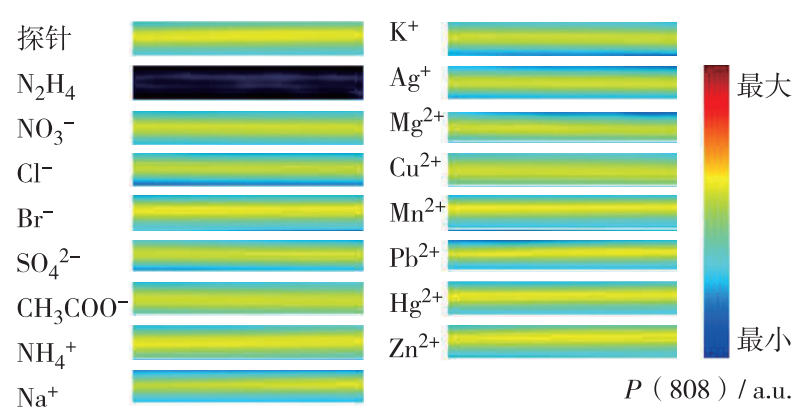

图 $8 \mathrm{Cy}-2$ 分别与肼、其他离子 $(100 \mu \mathrm{mol} / \mathrm{L})$ 作用后在 $808 \mathrm{~nm}$ 处光声信号变化

Fig. 8 PA images of $\mathrm{Cy}-2$ at $808 \mathrm{~nm}$ with $100 \mu \mathrm{mol} / \mathrm{L}$ of hydrazine and other ions

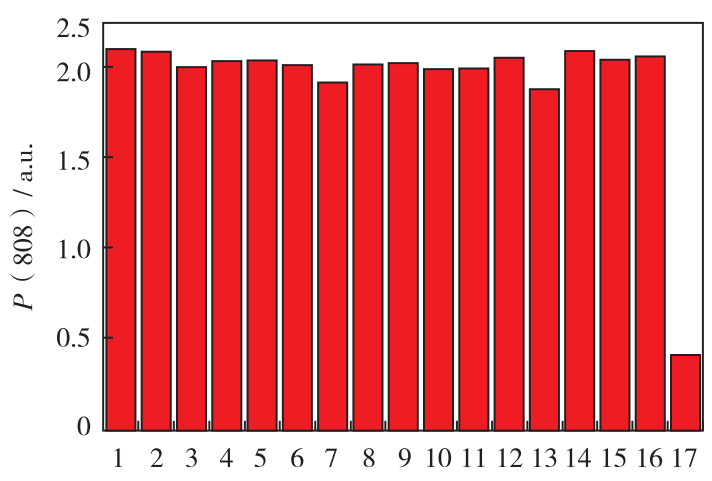

1 为 $\mathrm{Cy}-2 ; 2$ 为 $\mathrm{SO}_{4}{ }^{2-} ; 3$ 为 $\mathrm{NO}_{3}{ }^{-} ; 4$ 为 $\mathrm{Cl}^{-} ; 5$ 为 $\mathrm{Br}^{-}$; 6 为 $\mathrm{CH}_{3} \mathrm{COO}^{-} ; 7$ 为 $\mathrm{NH}_{4}^{+} ; 8$ 为 $\mathrm{Na}^{+} ; 9$ 为 $\mathrm{K}^{+} ; 10$ 为 $\mathrm{Ag}^{+}$;

11 为 $\mathrm{Zn}^{2+} ; 12$ 为 $\mathrm{Hg}^{2+} ; 13$ 为 $\mathrm{Mg}^{2+} ; 14$ 为 $\mathrm{Cu}^{2+}$; 15 为 $\mathrm{Mn}^{2+} ; 16$ 为 $\mathrm{Pb}^{2+} ; 17$ 为 $\mathrm{N}_{2} \mathrm{H}_{4}$

图 $9 \mathrm{Cy}-2$ 分别与肼、其他离子 $(100 \mu \mathrm{mol} / \mathrm{L})$ 作用后 $P(808)$ 定量值

Fig. 9 Quantized $P(808)$ after Cy-2 responding to $100 \mu \mathrm{mol} / \mathrm{L}$ of hydrazine and other ions

\section{结 语}

本研究设计并合成了一种以花菁类染料为母体 的光声分子探针 $\mathrm{Cy}-2$, 实现了肼的光声检测. 该探 针在波长 $808 \mathrm{~nm}$ 处具有较强的光声信号. 当探针
分子与肼作用后, 随着溶液中肼浓度的增高, 分子 探针在 $808 \mathrm{~nm}$ 处的光密度显著下降, 而 $550 \mathrm{~nm}$ 处 的光密度明显升高, 且在一定浓度范围内（ $0 \sim 10$ $\mu \mathrm{mol} / \mathrm{L}), D(808) / D(550)$ 与肼浓度具有良好的线 性关系. 同时, 新型分子探针 $808 \mathrm{~nm}$ 处的光声信 号强度也随着肼浓度增加而降低, 在肼浓度为 0 $10 \mu \mathrm{mol} / \mathrm{L}$ 内, $P(808)$ 与肼浓度呈良好的线性关 系, 肼检测限为 $0.50 \mu \mathrm{mol} / \mathrm{L}$. 该探针合成方法简 单, 并能够对肼实现快速高效的光声检测, 有望用 于在体或深层组织肼的检测.

致 谢：感谢深圳大学丽湖校区测试中心的支持！

\footnotetext{
基金项目: 国家重点基础研究发展计划资助项目（2018YFA0704 $003)$

作者简介：蒋 超 (1994-), 深圳大学硕士研究生. 研究方向: 分子影像学纳米医学. E-mail jiangchao2017@ email.szu.edu.cn

引文: 蒋 超, 林 静, 黄 鹏. 新型近红外花菁类光声探针 用于肼检测 $[\mathrm{J}]$. 深圳大学学报理工版, 2020,37 (1): 33-38.
}

\section{参考文献／References:}

[ 1 ] HU Lirong. Research on application of hydrazine hydrate as reductant in organic synthesis $[\mathrm{J}]$. Chemical Industry Times, 2012, 26(4) : 41-58.

[ 2 ] SELVAKUMAR S, SOMANATHAN N, REDDY $\mathrm{K}$ A. Chemiresistor sensors based on conducting polymers for hypergolic propellants and acidic vapors of rocket exhaust plumes: a review $[\mathrm{J}]$. Propellants Explosives Pyrotechnics, 2013, 38(2) : 176-189.

[ 3 ] REILLY C A, AUST S D. Peroxidase substrates stimulate the oxidation of hydralazine to metabolites which cause single-strand breaks in DNA [J]. Chemical Research in Toxicology, 1997, 10(3) : 328-334.

[ 4 ] GARROD S, BOLLARD M E, NICHOLLST A W, et al. Integrated metabonomic analysis of the multiorgan effects of hydrazine toxicity in the rat $[\mathrm{J}]$. Chemical Research in Toxicology, 2005, 18(2): 115-122.

[ 5 ] SULTANA W, ERAIAH B, VASAN H N. Efficient polyglycine modified Au electrode for the detection of hydrazine $[\mathrm{J}]$. Analytical Methods, 2012, 4 ( 12 ): 4115-4120.

[ 6 ] ABDUL AZIZ M, KAWDE A N. Gold nanoparticle-modified graphite pencil electrode for the high-sensitivity detection of hydrazine $[\mathrm{J}]$. Talanta, 2013(115) : 214-221.

[ 7 ] WANG J, CHEN Liang. Hydrazine detection using a tyrosinase-based inhibition biosensor [ J ]. Analytical Chemistry, 1995, 67(20): 3824-3827. 
[ 8 ] MCBRIDE W R, HENRY R A, SKOLNIK S. Potentiometric titration of organic derivatives of hydrazine with potassium iodate $[\mathrm{J}]$. Analytical Chemistry, 1953, 25 (7) : 1042-1046.

[ 9 ] GOSWAMI S, AICH K, DAS S, et al. A reaction based colorimetric as well as fluorescence 'turn on' probe for the rapid detection of hydrazine $[\mathrm{J}]$. RSC Advances, 2014, 4(27) : 14210-14214.

[10 ] LI Biao, HE Zhaoshuai, ZHOU Hanxin, et al. Reaction based colorimetric and fluorescence probes for selective detection of hydrazine $[\mathrm{J}]$. Dyes and Pigments, 2017 (146) : 300-304.

[11] YU Fabiao, LI Peng, SONG Ping, et al. An ICT-based strategy to a colorimetric and ratiometric fluorescence probe for hydrogen sulfide in living cells $[\mathrm{J}]$. Chemical Communications, 2012, 48(23) : 2852-2854.

[12] CHEN Xiaoqiang, TIAN Xizhe, SHIN I, et al. Fluorescent and luminescent probes for detection of reactive oxygen and nitrogen species $[\mathrm{J}]$. Chemical Society Reviews, 2011, 40(9): 4783-4804.

[13] FRANGIONI J V. In vivo near-infrared fluorescence imaging $[\mathrm{J}]$. Current Opinion in Chemical Biology, $2003,7(5): 626-634$.

[14] XU Jingjuan, ZHAO Weiwei, SONG Shiping, et al. Functional nanoprobes for ultrasensitive detection of biomolecules: an update $[\mathrm{J}]$. Chemical Society Reviews, 2014, 43(5) : 1601-1611.

[15] ZENG Leli, MA Gongcheng, LIN Jing, et al. Photoacoustic probes for molecular detection: recent advances and perspectives [J]. Small, 2018, 14(30): e1800782.

[16] WANG Sheng, LIN Jing, WANG Tianfu, et al. Recent advances in photoacoustic imaging for deep-tissue biomedical applications [ J ]. Theranostics, 2016, 6 (13) : 2394-2413.

[17] XIE Chen, ZHEN Xu, LYU Yan, et al. Nanoparticle regrowth enhances photoacoustic signals of semiconducting macromolecular probe for in vivo imaging $[\mathrm{J}]$. Advanced Materials, 2017, 29(44): 1703693

[18 ] LIU Yajing, LIU Huanhuan, YAN Huixiang, et al. Aggregation-induced absorption enhancement for deep near-infrared II photoacoustic imaging of brain gliomas in vivo $[\mathrm{J}]$. Advanced Science, 2019, 6(8) : 1801615.

[19] LIU Yajing, YANG Yanping, SUN Mingiian, et al. Highly specific noninvasive photoacoustic and positron emission tomography of brain plaque with functionalized croconium dye labeled by a radiotracer $[\mathrm{J}]$. Chemical Science, $2017,8(4)$ : 2710-2716.
[20 ] LI Weitao, CHEN Ronghe, LV ( LÜ) Jing, et al. In vivo photoacoustic imaging of brain injury and rehabilitation by high-efficient near-infrared dye labeled mesen-chymal stem cells with enhanced brain barrier permeability $[\mathrm{J}]$. Advanced Science, 2018, 5(2): 1700277.

[21] WANG Sheng, LIN Jing, WANG Zhantong, et al. Coresatellite polydopamine-gadolinium-metallofullerene nanotheranostics for multimodal imaging guided combination cancer therapy $[\mathrm{J}]$. Advanced Materials, 2017, 29 (35) : 1701013.

[22] LI Chunxiao, ZHANG Yifan, LI Zhiming, et al. Lightresponsive biodegradable nanorattles for cancer theranostics [J]. Advanced Materials, 2018, 30: 1870049.

[23] QIN Xialing, LI Fan, ZHANG Yifan, et al. In vivo photoacoustic detection and imaging of peroxynitrite $[\mathrm{J}]$. Analytical Chemistry, 2018, 90(15) : 9381-9385.

[24] HUANG Yan, LI Fan, MA Gongcheng, et al. Aggregation induced photoacoustic detection of mercury ( II) ions using quaternary ammonium group-capped gold nanorods $[\mathrm{J}]$. Talanta, 2018, 187: 65-72.

[25] LIU Yi, WANG Sheng, MA Ying, et al. Ratiometric photoacoustic molecular imaging for methylmercury detection in living subjects $[\mathrm{J}]$. Advanced Materials, $2017,29(17)$ : 1606129.

[26] ZENG Leli, MA Gongcheng, XU Han, et al. In vivo chemoselective photoacoustic imaging of copper ( II ) in plant and animal subjects $[\mathrm{J}]$. Small, 2019, $15(6)$ : e1803866.

[27] MA Gongcheng, GAO Xiaoting, JIANG Chao, et al. pHResponsive nanoprobe for in vivo photoacoustic imaging of gastric acid [J]. Analytical Chemistry, 2019, 91(21) : 13570-13575.

[28 ] GAO Xiaoting, MA Gongcheng, JIANG Chao, et al. In vivo near-infrared fluorescence and photoacoustic dualmodal imaging of endogenous alkaline phosphatase $[\mathrm{J}]$. Analytical Chemistry, 2019, 91(11) : 7112-7117.

[29] LI Lei, SHEMETOV A A, BALOBAN M, et al. Small near-infrared photochromic protein for photoacoustic multicontrast imaging and detection of protein interactions in vivo $[\mathrm{J}]$. Nature Communications, 2018, 9 ( 1): 2734.

[30 ] MÄRK J, DORTAY H, WAGENER A, et al. Dualwavelength 3D photoacoustic imaging of mammalian cells using a photoswitchable phytochrome reporter protein [J]. Communications Physics, 2018, 1(1): 3 .

【中文责编：晨 兮; 英文责编：新 谷】 